\title{
Weathering effects on engineering properties of Schist of Menderes Massif, West of Turkey
}

Berna Şengöçmen Geçkin ( $\square$ bernasengocmen@gmail.com )

Science Institute of Dokuz Eylul https://orcid.org/0000-0003-3473-5665

\section{Necdet Turk}

Science Institute of Dokuz Eylul University

\section{Dyson Moses}

Kyushu University, Department of Earth Resource Engineering

\section{Research Article}

Keywords: Menderes Massive, Schist, Weathering, XRF, Porosity, Water content

Posted Date: February 3rd, 2021

DOl: https://doi.org/10.21203/rs.3.rs-177549/v1

License: (1) This work is licensed under a Creative Commons Attribution 4.0 International License. Read Full License 


\section{Abstract}

Schist is a metamorphic rock type that is widely exposed in the Western Anatolia. Schist of Menderes Massif is located in different locations and geological levels. The rock is mainly used as a filling and building material in the present and in foreseeable future. Usage of schist as filling materials in dams is related with their weathering degree which affects their geomechanical properties. To determinate the petrographic, chemical, index and engineering properties of the schist used as filler material in the dams/small dam crest, fresh and weathered samples of rock material were collected from quarries. The relationship between the weathering degree index and engineering properties were determined by simple regression analyses'. The results show that the studied parameters of physical and mechanical properties of the studied schist have a positive correction with weathering grade. Thus, increasing weathering grade affects the durability of the schist thereby compromising its applicability.

\section{Full Text}

This preprint is available for download as a PDF.

\section{Tables}

Table 1 Summary of weathering indices evaluated in this study 


\begin{tabular}{|c|c|c|c|c|c|}
\hline $\begin{array}{l}\text { Weathering } \\
\text { Index }\end{array}$ & Symbol & Formula & $\begin{array}{c}\text { Value in the } \\
\text { Unweath. } \\
\text { Rock }\end{array}$ & $\begin{array}{c}\text { Value in the } \\
\text { Weath. } \\
\text { Rock }\end{array}$ & Reference \\
\hline $\begin{array}{c}\text { Weathering } \\
\text { Index of Parker }\end{array}$ & WIP & $\begin{array}{c}\mathrm{CIA}=100 \times(\mathrm{Al} 2 \mathrm{O} 3 /(\mathrm{Al} 2 \\
\mathrm{O} 3+\mathrm{CaO}+\mathrm{Na} 2 \mathrm{O}+\mathrm{K} 2 \mathrm{O}))\end{array}$ & $>100$ & 0 & [23] \\
\hline $\begin{array}{c}\text { Chemical } \\
\text { Alteration Index }\end{array}$ & CIA & $\begin{array}{c}\mathrm{CIA}=100 \times(\mathrm{Al} 2 \mathrm{O} 3 /(\mathrm{Al} 2 \\
\mathrm{O} 3+\mathrm{CaO}+\mathrm{Na} 2 \mathrm{O}+\mathrm{K} 2 \mathrm{O}))\end{array}$ & $\leq 50$ & 100 & {$[24]$} \\
\hline $\begin{array}{l}\text { Chemical Index } \\
\text { of Weathering }\end{array}$ & CIW & $\begin{array}{c}\mathrm{CIW}=100 \mathrm{X}(\mathrm{Al} 2 \mathrm{O} 3 /((\mathrm{Al} 2 \mathrm{O} 3+\mathrm{CaO}+ \\
\mathrm{Na} 2 \mathrm{O}))\end{array}$ & $\leq 50$ & 100 & [22] \\
\hline $\begin{array}{l}\text { Alteration Index } \\
\text { of Plagioclase }\end{array}$ & PIA & $\begin{aligned} \mathrm{PIA}= & 100 \mathrm{X}((\mathrm{Al} 2 \mathrm{O} 3-\mathrm{K} 2 \mathrm{O}) /(\mathrm{Al} 2 \\
& \mathrm{O} 3+\mathrm{CaO}+\mathrm{Na} 2 \mathrm{O}-\mathrm{K} 2 \mathrm{O}))\end{aligned}$ & $\leq 50$ & 100 & {$[25]$} \\
\hline $\begin{array}{l}\text { Weathering } \\
\text { Index }\end{array}$ & WI & $\mathrm{WI}=100 \mathrm{X}(\mathrm{LOI} /(\mathrm{CaO}+\mathrm{MgO}+\mathrm{MnO}))$ & & & [17] \\
\hline Ruxtion Ratio & $\mathrm{RR}$ & $\mathrm{RR}=100 \mathrm{X}(\mathrm{SiO} 2 /(\mathrm{Al} 2 \mathrm{O} 3))$ & $<2.9$ & $>2.9$ & [18] \\
\hline $\begin{array}{l}\text { Silica-Titania } \\
\text { Index }\end{array}$ & STI & $\begin{array}{c}\mathrm{STI}=100 \times[\mathrm{SiO} 2 /((\mathrm{Ti} \mathrm{O} 2)] /[\mathrm{SiO} 2 /(\mathrm{Ti} \mathrm{O} 2 \\
)+\mathrm{SiO} 2 /(\mathrm{Al} 2 \mathrm{O} 3)+(\mathrm{Al} 2 \mathrm{O} 3) /(\mathrm{Ti} \mathrm{O} 2)]\end{array}$ & $>90$ & 0 & {$[26]$} \\
\hline
\end{tabular}

Table 2 Mineral contents of rocks observed in the study areas 


\begin{tabular}{|c|c|c|c|c|c|c|c|c|}
\hline & \multirow[b]{2}{*}{ Rock Material } & \multicolumn{7}{|c|}{ Minerals } \\
\hline & & $\begin{array}{c}\text { Quartz } \\
\%\end{array}$ & Calcite $\%$ & $\begin{array}{c}\text { Musquovite } \\
\%\end{array}$ & $\begin{array}{c}\text { Graphite } \\
\%\end{array}$ & $\begin{array}{c}\text { Feldspar } \\
\%\end{array}$ & $\begin{array}{c}\text { Garnet } \\
\%\end{array}$ & $\begin{array}{l}\text { Chlorite+ } \\
\text { Biotite \% }\end{array}$ \\
\hline \multirow{3}{*}{ 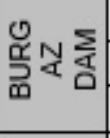 } & Musquovite Calcschist & 10 & 60 & 30 & - & - & - & - \\
\hline & Graphite Calcschist & - & 60 & 10 & 30 & - & - & - \\
\hline & Quartz Calcschist & 20 & 50 & 10 & 10 & - & - & - \\
\hline \multirow{5}{*}{ 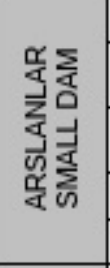 } & Musquovite Calcschist & 50 & 20 & 30 & - & - & - & - \\
\hline & $\begin{array}{c}\text { Musquovite Schist with } \\
\text { Garnet }\end{array}$ & 40 & - & 30 & - & 10 & 10 & 10 \\
\hline & $\begin{array}{c}\text { Biotite-Musquovite Schist } \\
\text { with Garnet }\end{array}$ & 30 & - & 10 & - & 10 & 20 & 30 \\
\hline & Musquovite Quartzschist & 80 & - & 20 & - & - & - & - \\
\hline & Musquovite Quartzschist & 80 & - & 20 & - & - & - & - \\
\hline \multirow{6}{*}{ 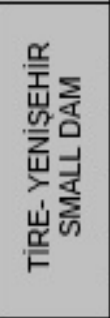 } & $\begin{array}{c}\text { Biotite-Musquovite Schist } \\
\text { with Garnet }\end{array}$ & 40 & - & 20 & - & - & 20 & 20 \\
\hline & Garnet Biotite Schist & 40 & - & - & - & - & 20 & 40 \\
\hline & $\begin{array}{c}\text { Biotite-Musquovite Schist } \\
\text { with Garnet }\end{array}$ & 30 & - & 20 & - & - & 20 & 30 \\
\hline & Chlorite Biotiteschist & 20 & 20 & - & - & - & - & 60 \\
\hline & Quartz-Musquoviteschist & 40 & - & 60 & - & - & - & - \\
\hline & Garnet Biotite Schist & 30 & - & - & - & - & 20 & 50 \\
\hline
\end{tabular}

Table 3 Major element composition of Menderes Massif Schist 


\begin{tabular}{|c|c|c|c|c|c|c|c|c|c|c|c|c|}
\hline & $\mathrm{Na}_{2} \mathrm{O}$ & $\mathrm{K}_{2} \mathrm{O}$ & $\sum_{\mathrm{Fe}_{2} \mathrm{O}_{3}}^{\sum}$ & $\mathrm{MnO}$ & $\mathrm{SiO}_{2}$ & $\mathrm{Al}_{2} \mathrm{O}_{2}$ & $\mathrm{CaO}$ & $\mathrm{MgO}$ & $\mathrm{TiO}_{2}$ & LOI & $\begin{array}{l}\text { Mean } \\
\text { value } \\
\text { of LOI }\end{array}$ & TOTAL \\
\hline Arslanlar Run 1 & 2.44 & 3.14 & 7.72 & 0.08 & 63.29 & 16.45 & 1.16 & 2.70 & 0.22 & 2.79 & \multirow{5}{*}{$\begin{array}{c}0.72 \\
\pm 0.12\end{array}$} & 99.98 \\
\hline Arslanlar Run 2 & 2.14 & 3.45 & 7.14 & 0.09 & 63.97 & 16.10 & 1.25 & 2.28 & 0.36 & 2.73 & & 99.52 \\
\hline Arslanlar Run 3 & 2.73 & 3.26 & 7.07 & 0.03 & 63.96 & 16.62 & 1.17 & 2.66 & 0.07 & 2.77 & & 100.34 \\
\hline Arslanlar Run 4 & 2.00 & 3.27 & 7.64 & 0.09 & 63.36 & 16.55 & 1.21 & 2.87 & 0.88 & 2.11 & & 100.40 \\
\hline Arslanlar Run 5 & 3.42 & 2.90 & 7.44 & 0.17 & 58.92 & 18.78 & 2.40 & 2.47 & 1.25 & 1.86 & & 100.30 \\
\hline Arslanlar Fun 1 & 0.37 & 0.52 & 1.40 & 0.04 & 92.09 & 3.48 & 0.91 & 0.27 & 0.19 & 0.70 & \multirow{8}{*}{$\begin{array}{c}2.45 \\
\pm 0.44\end{array}$} & 99.96 \\
\hline Arslanlar Fun 2 & 0.33 & 0.50 & 1.35 & 0.03 & 92.19 & 3.43 & 0.94 & 0.24 & 0.17 & 0.60 & & 99.78 \\
\hline Arslanlar Fun 3 & 0.34 & 0.55 & 1.32 & 0.04 & 92.35 & 3.55 & 0.88 & 0.21 & 0.15 & 0.62 & & 99.99 \\
\hline Arslanlar Fun 4 & 0.43 & 0.51 & 1.34 & 0.03 & 93.15 & 3.10 & 0.09 & 0.21 & 0.36 & 0.69 & & 99.96 \\
\hline Arslanlar Fun 5 & 0.51 & 0.59 & 1.11 & 0.06 & 90.26 & 3.89 & 0.80 & 0.15 & 0.96 & 0.91 & & 99.23 \\
\hline Arslanlar Fun 6 & 0.52 & 0.58 & 1.19 & 0.06 & 90.23 & 3.88 & 0.74 & 0.11 & 0.98 & 0.73 & & 99.02 \\
\hline Arslanlar Fun 7 & 0.51 & 0.59 & 1.18 & 0.06 & 90.75 & 3.82 & 0.72 & 0.12 & 0.97 & 0.88 & & 99.60 \\
\hline Arslanlar Fun 8 & 0.50 & 0.57 & 1.65 & 0.03 & 91.78 & 3.33 & 0.07 & 0.21 & 0.38 & 0.65 & & 99.29 \\
\hline Burgaz 1 & 2.83 & 2.14 & 5.87 & 0.12 & 68.60 & 14.24 & 1.89 & 1.73 & 0.29 & 1.87 & \multirow{8}{*}{$\begin{array}{c}2.06 \\
\pm 0.26\end{array}$} & 99.57 \\
\hline Burgaz 2 & 3.00 & 2.71 & 5.81 & 0.12 & 68.01 & 14.40 & 1.88 & 1.73 & 0.27 & 1.82 & & 99.74 \\
\hline Burgaz 3 & 2.53 & 2.43 & 5.91 & 0.12 & 68.59 & 14.34 & 1.90 & 1.71 & 0.47 & 1.89 & & 99.89 \\
\hline Burgaz 4 & 2.80 & 2.14 & 5.62 & 0.11 & 67.51 & 14.44 & 1.84 & 1.75 & 0.85 & 1.86 & & 99.42 \\
\hline Burgaz 5 & 2.53 & 2.58 & 7.54 & 0.06 & 65.50 & 15.03 & 1.16 & 2.51 & 0.27 & 2.30 & & 99.48 \\
\hline Burgaz 6 & 2.97 & 3.00 & 7.74 & 0.08 & 63.89 & 15.50 & 1.25 & 2.53 & 0.47 & 2.57 & & 100.00 \\
\hline Burgaz 7 & 2.53 & 2.45 & 7.64 & 0.06 & 65.38 & 15.42 & 1.17 & 2.13 & 0.20 & 2.13 & & 99.11 \\
\hline Burgaz 8 & 2.51 & 3.60 & 7.88 & 0.07 & 62.10 & 15.29 & 1.67 & 2.94 & 1.07 & 2.04 & & 99.25 \\
\hline Yenişehir 1 & 1.51 & 3.64 & 6.95 & 0.10 & 63.76 & 17.56 & 0.50 & 0.57 & 0.41 & 4.32 & \multirow{8}{*}{$\begin{array}{c}4.08 \\
\pm 0.25\end{array}$} & 99.33 \\
\hline Yenişehir 2 & 1.59 & 3.62 & 6.91 & 0.11 & 63.81 & 17.40 & 0.44 & 0.53 & 0.41 & 4.38 & & 99.20 \\
\hline Yenişehir 3 & 1.56 & 3.61 & 6.98 & 0.12 & 63.81 & 17.39 & 0.42 & 0.51 & 0.45 & 4.36 & & 99.21 \\
\hline Yenişehir 4 & 1.43 & 3.74 & 6.85 & 0.08 & 63.75 & 17.66 & 0.46 & 0.57 & 0.85 & 4.11 & & 99.84 \\
\hline Yenişehir 5 & 1.43 & 3.20 & 7.73 & 0.09 & 63.65 & 17.66 & 0.39 & 0.76 & 0.43 & 3.86 & & 99.18 \\
\hline Yenişehir 6 & 1.45 & 3.12 & 7.85 & 0.10 & 63.62 & 17.93 & 0.18 & 0.70 & 0.41 & 3.75 & & 99.12 \\
\hline Yenişehir 7 & 1.38 & 3.04 & 7.63 & 0.10 & 63.61 & 17.99 & 0.70 & 0.65 & 0.45 & 3.86 & & 99.41 \\
\hline Yenişehir 8 & 1.60 & 3.65 & 7.17 & 0.07 & 63.34 & 17.65 & 0.49 & 0.68 & 0.84 & 3.99 & & 99.81 \\
\hline
\end{tabular}

Table 4 Results of the weathering grades of the analyzed samples

\begin{tabular}{ccc}
\hline Dam/Small Dam & $\begin{array}{c}\text { Water Content (\%) } \\
\text { (Min-Max) }\end{array}$ & Weathering Grade \\
\hline Arslanlar Small Dam - Fun Rock Material Quarry \\
$\begin{array}{c}\text { Quartzschist } \\
\text { Arslanlar Small Dam - Run Rock Material Quarry } \\
\text { Musquoviteschist }\end{array}$ & $0.51-0.90$ & W1 \\
\hline $\begin{array}{c}\text { Burgaz Dam - Rock Material Quarry } \\
\text { Calcshist }\end{array}$ & $2.89-2.80$ & W3 \\
\hline $\begin{array}{c}\text { Yenişehir Small Dam - Rock Material Quary } \\
\text { Biotiteschist }\end{array}$ & $1.20-4.41$ & W3
\end{tabular}

Table 5 Physical and mechanical properties of studied schists at various degree of weathering 


\begin{tabular}{|c|c|c|c|c|c|c|c|c|c|c|c|}
\hline & & & $\begin{array}{c}7 / d \\
\left(9 / \mathrm{cm}^{3}\right)\end{array}$ & $\begin{array}{c}n \\
(\%)\end{array}$ & $V p(m / s n)$ & $\begin{array}{c}\mathrm{Na}_{2} \mathrm{SO}_{4} \\
\text { freeze- } \\
\text { thawi } \\
\text { durability } \\
(\%)\end{array}$ & $\begin{array}{c}\text { Los Angeles } \\
\text { test } \\
(\mathrm{LA}, \%)\end{array}$ & $\begin{array}{c}\text { Slake } \\
\text { Durability } \\
\text { (SD, \%) }\end{array}$ & $\begin{array}{c}\text { Uniaxial } \\
\text { Comp. } \\
\text { Strength } \\
\text { (UCS, MPa) }\end{array}$ & $\begin{array}{c}\text { Point } \\
\text { Load } \\
\text { Strength } \\
\left(\mathrm{Is}_{50}\right. \\
\mathrm{MPa}) \\
\end{array}$ & $\begin{array}{l}\text { Brazilian } \\
\text { Tensile } \\
\text { Strength } \\
\text { (TS,MPa) }\end{array}$ \\
\hline Dam & Rock Type & WG & $\begin{array}{l}\text { TS EN } \\
1097-6 \\
\end{array}$ & $\begin{array}{l}\text { ISRM } \\
1981 \\
\end{array}$ & ISRM 1981 & ASTM C33 & $\begin{array}{c}\text { TS EN-1097- } \\
2 \\
\end{array}$ & ISRM 1981 & ISRM 1981 & ISRM 1985 & ISRM 1981 \\
\hline $\begin{array}{l}\text { Arslanlar } \\
\text { Small } \\
\text { Dam Fun } \\
\text { Arslanlar }\end{array}$ & Quartzschist & W1 & $2.71 \pm 0.3$ & $0.75 \pm 0.2$ & $4500 \pm 300$ & $1.85 \pm 0.1$ & $27.60 \pm 0.1$ & $98.90 \pm 0.1$ & $47.04 \pm 10.06$ & $6.20 \pm 1.10$ & $3.50 \pm 0.40$ \\
\hline $\begin{array}{l}\text { Small } \\
\text { Dam } \\
\text { Run }\end{array}$ & Musquoviteschist & w3 & $2.67 \pm 0.1$ & $2.35 \pm 0.5$ & $2709 \pm 248$ & $3.14 \pm 0.1$ & $46.34 \pm 0.1$ & $97.20 \pm 0.1$ & $23.66 \pm 6.60$ & $4.80 \pm 0.10$ & $1.50 \pm 0.80$ \\
\hline $\begin{array}{c}\text { Burgaz } \\
\text { Dam } \\
\text { Yenișehir }\end{array}$ & Calcshist & w3 & $2.61 \pm 0.2$ & $2.44 \pm 0.3$ & $3455 \pm 1902$ & $4.72 \pm 0.1$ & $47.50 \pm 0.1$ & $97.78 \pm 0.1$ & $32.80 \pm 9.12$ & $2.45 \pm 0.80$ & $2.08 \pm 0.77$ \\
\hline $\begin{array}{l}\text { Small } \\
\text { Dam }\end{array}$ & Biotiteschist & w4 & $2.56 \pm 0.8$ & $2.81 \pm 1.6$ & $2824 \pm 1430$ & $5.36 \pm 1.78$ & $49.88 \pm 26.41$ & $97.97 \pm 0.1$ & $21.30 \pm 1.20$ & $1.50 \pm 0.30$ & $1.42 \pm 0.30$ \\
\hline
\end{tabular}

$W G$ : Weathering Grade; $n:$ Porosity; $g_{d}$ : Dry density; $V p$ : Ultrasonic velocity

Table 6 The best correlations between loss on ignition and mechanical and physical properties of the studied schist

\begin{tabular}{lll}
\hline Variables & Formula & $r$ \\
\hline UCS vs. LOI & UCS $=-15.553 \mathrm{Ln}(\mathrm{LOI})+41.696$ & 0.97 \\
$\mathrm{n}(\%)$ vs. LOI & $\mathrm{n}=1.2233 \mathrm{LN}(\mathrm{LOI})+1.2619$ & 0.97 \\
$\mathrm{~V} p(\mathrm{~m} / \mathrm{sn})$ vs LOI & $\mathrm{V} p=-1053.8 \mathrm{Ln}(\mathrm{LOI})+4083.2$ & 0.93 \\
$\mathrm{Na} 2 \mathrm{SO}_{4}(\%)$ vs LOI & $\mathrm{Na} 2 \mathrm{SO} 4=2.3349(\mathrm{LOI})^{0.5921}$ & 0.89 \\
$\mathrm{SD}(\%)$ vs LOI & $\mathrm{SD}=98.432 \mathrm{LOI}-0.0071$ & 0.72 \\
$\mathrm{LA}(\%)$ vs. LOI & $\mathrm{LA}=13.48 \mathrm{Ln}(\mathrm{LOI})+33.733$ & 0.97 \\
Is50 $(\mathrm{MPa})$ vs LOI & $\mathrm{Is} 50=-2.4731 \mathrm{Ln}(\mathrm{LOI})+5.4065$ & 0.84 \\
$\mathrm{TS}(\mathrm{MPa})$ vs LOI & $\mathrm{TS}=-1.2804 \mathrm{Ln}(\mathrm{LOI})+2.9891$ & 0.97
\end{tabular}

\section{Declarations}

The academic conflict of interest lists is given below.

Atiye Tuğrul Prof., Istanbul Universitesi tugrul@istanbul.edu.tr She has researched in a smilar topic

Şener Ceryan, Dr Lecturer, Balıkesir Universitesi sceryan@balikesir.edu.tr He has researched the same of topic

\section{Figures}




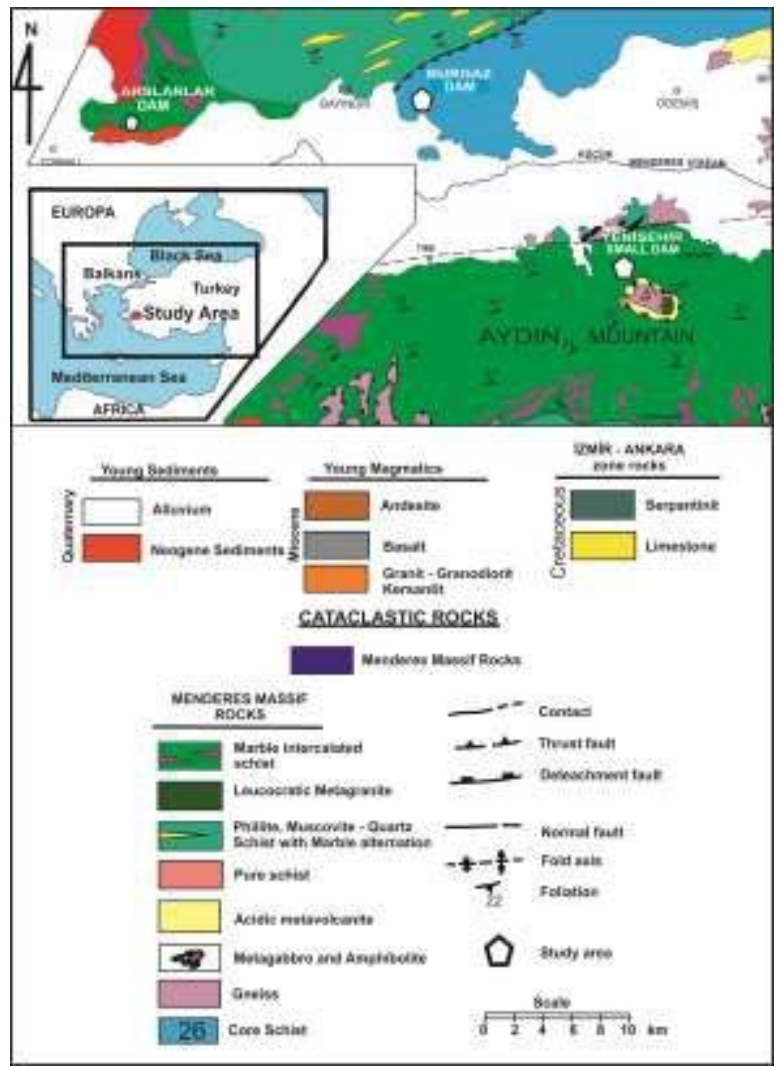

Figure 1

Locations of the quarries where samples were taken within the scope of the research (From left to right Aslanlar dam, Burgaz Dam and Yenişehir Dam, respectively)
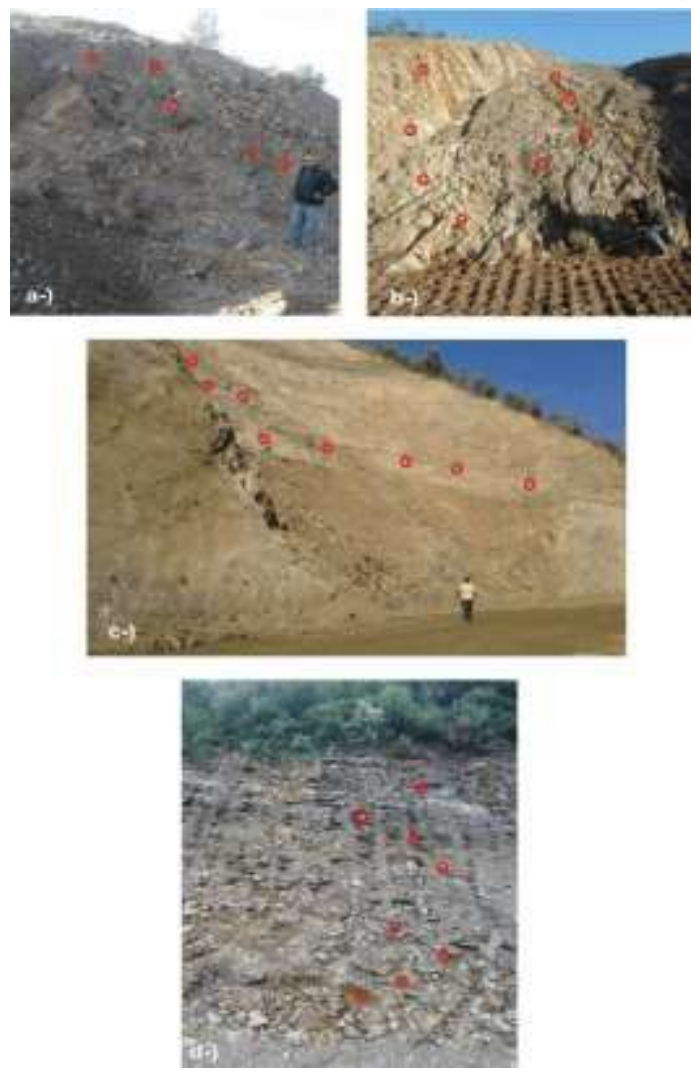
Figure 2

Sampling areas a-) Arslanlar small dam-quarry run rockfill material b-) Arslanlar small damquarry fun rockfill material c-) Burgaz dam- rock material quarry d-)Yenişehir small dam- rock material
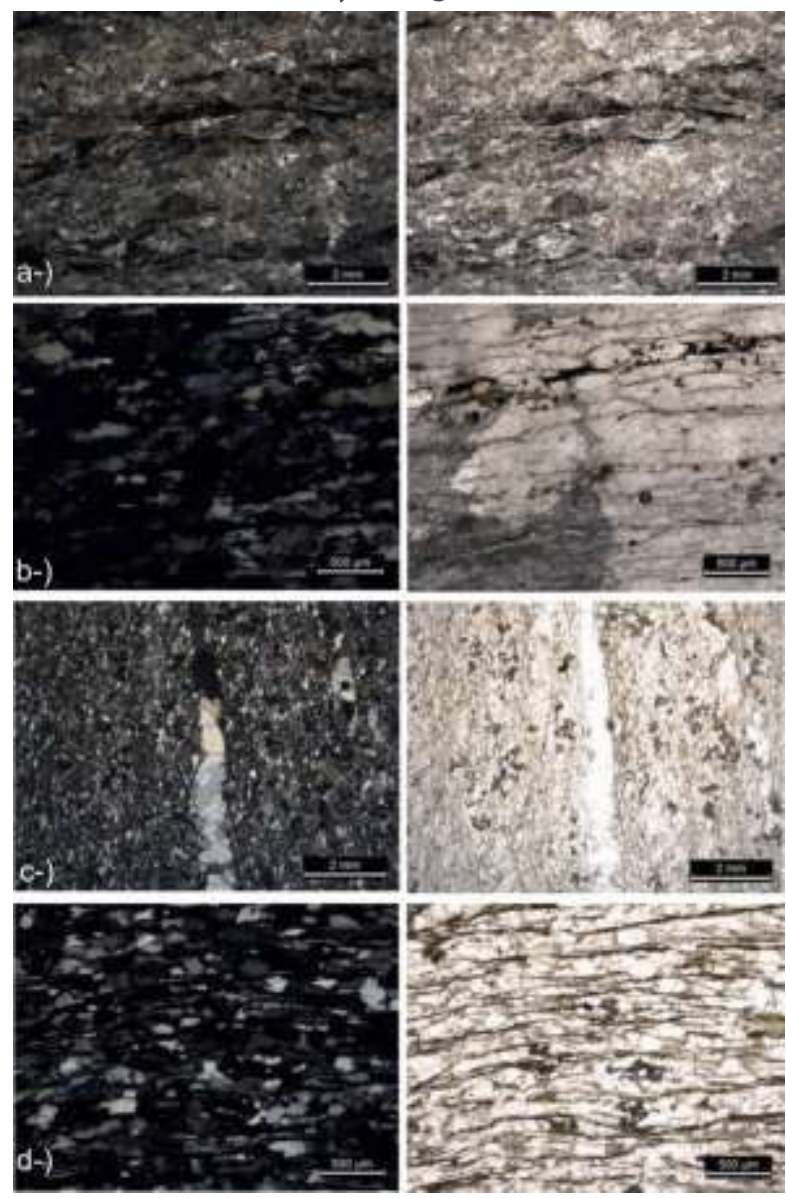

\section{Figure 3}

Thin section images of a-) Burgaz dam b-) and c-) Arslanlar small dam fun and run rock material quarry, respectively $d-$ ) Yenişehir small dam rock material quarry 

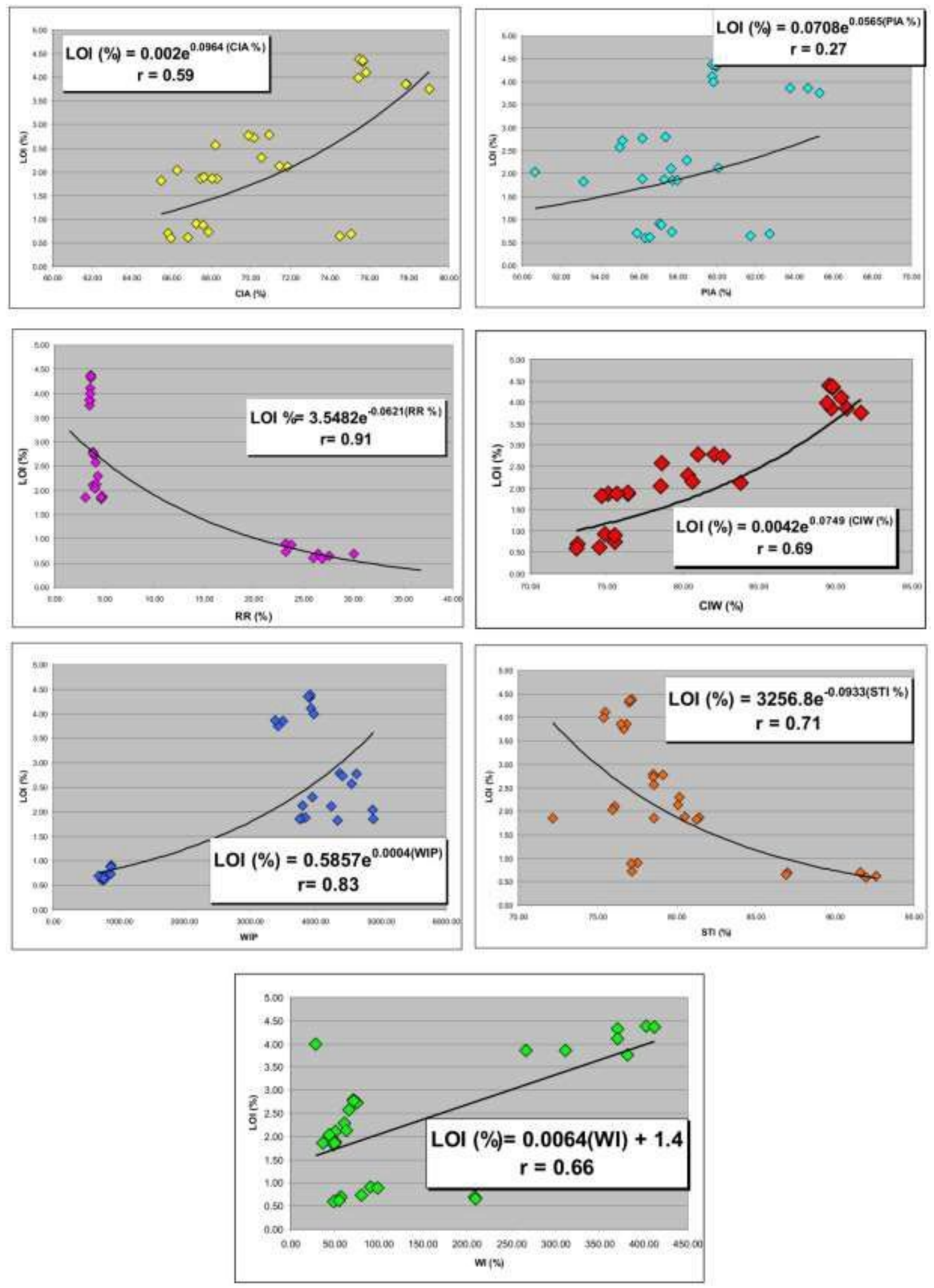

\section{Figure 4}

The relationship between LOI and weathering indices 

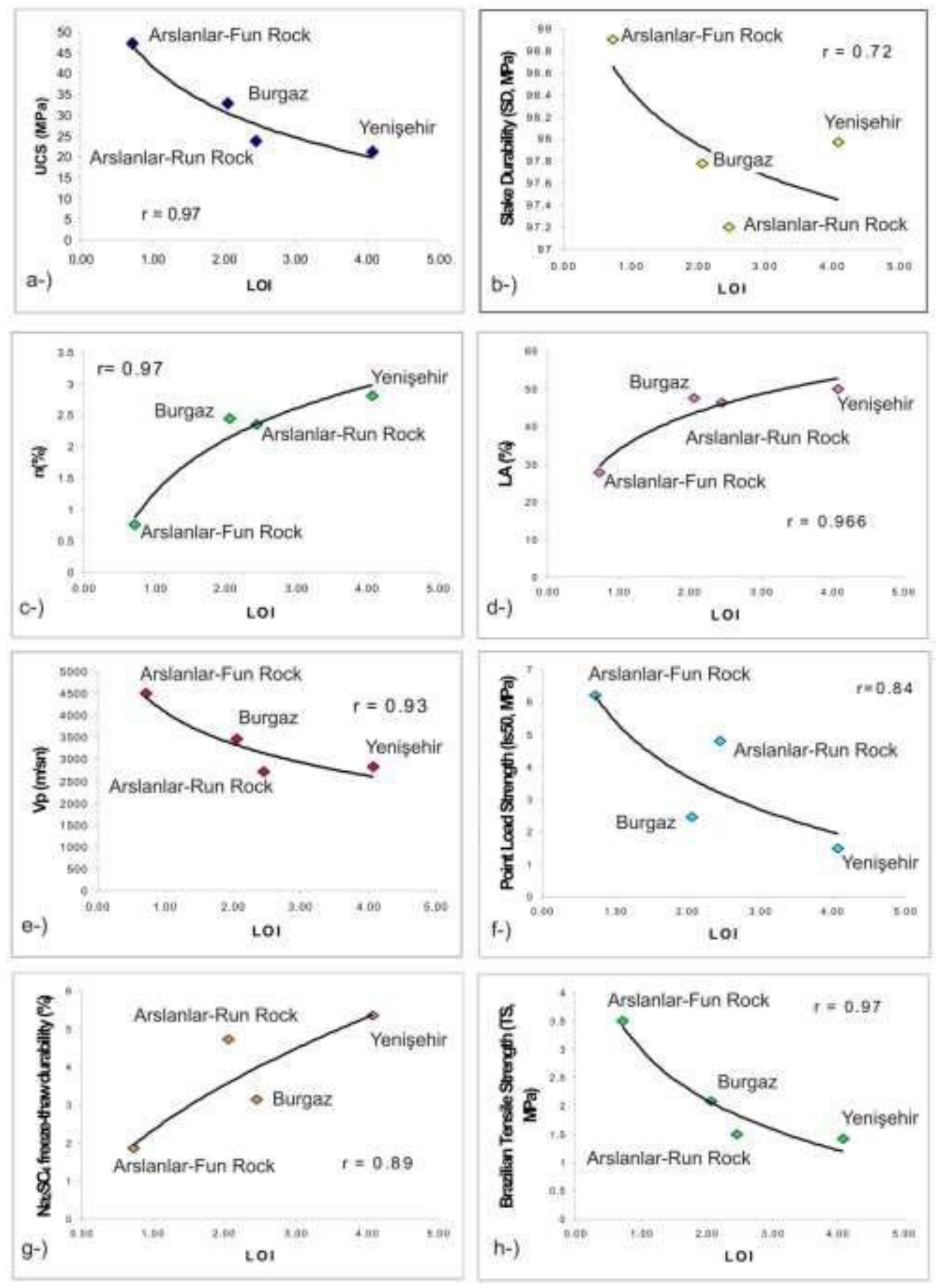

\section{Figure 5}

Relationship between mean value of LOI and physical and mechanical properties of tested schists a-) UCS vs LOI; b-) SD vs. LOl; c-) n vs LOI; d-) LA vs. LOI; e-) Vp vs LOl; f-)Is50 vs LOl; g-) Na2SO4 vs. LOl; h-) TS vs LOI 